\title{
LIGHT PROPAGATION IN A CLUMPY UNIVERSE
}

\author{
LAM HUI AND UROŠ SELJAK \\ Department of Physics \\ Massachusetts Institute of Technology \\ Cambridge, Massachusetts 02139 USA.
}

The propagation of light in an inhomogeneous universe is a long standing problem. Its resolution requires, first, a realistic description of the geometry of a clumpy universe and, second, solutions to the null geodesic equations given the metric of such a universe. The Friedmann-Robertson-Walker metric has become the standard description of the large scale geometry of the universe. However, the observable universe today is manifestly inhomogeneous. The weakly perturbed Friedmann-Robertson-Walker metric is often used to describe such a universe. But its validity is only guaranteed for a weakly inhomogeneous universe, where, for instance, overdensities are small $(\delta \rho / \bar{\rho} \ll 1)$, which is not true for sufficiently small scales in the universe today. It is well known, however, that the metric perturbations can still be small even if the overdensity is not small, given the right conditions and coordinates. However, spatial gradients of metric perturbations are not necessarily small any more. Here we estimate whether the secondorder corrections involving them can affect significantly the expansion of the universe or the light propagation in it.

We concentrate on the energy constraint equation (or the time-time part of the Einstein's equations). The zeroth order terms of this equation constitute the Friedmann equation. The second order terms that we keep are then corrections to it. They can be viewed as the back-reaction of inhomogeneities on the expansion rate of the universe (Futamase 1988). We write the metric in the following form:

$$
d s^{2}=a^{2}(\eta)\left[-(1+2 \phi) d \eta^{2}+2 \omega_{i} d x^{i} d \eta+\left((1-2 \psi) \delta_{i j}+2 h_{i j}\right) d x^{i} d x^{j}\right]
$$

where $\phi, \omega_{i}, \psi$ and $h_{i j}$ are functions of time and space. $h_{i j}$ is chosen to be traceless i.e. $\sum_{k} h_{k k}=0$. We also make the slow motion approximation: peculiar velocities are small and so are the time derivatives of metric perturbations compared to their gradients. We impose the gauge conditions 
$\omega^{i}{ }_{i}=0$ and $h_{i j}{ }^{i}=0$. By examining the rest of the Einstein equations, it can be shown that $\omega_{i}$ and $h_{i j}$ and their gradients are small compared to $\phi$ or $\psi$. So, second order terms involving them are discarded. All other second order terms involving spatial gradients are kept. The time-time equation can be separated into homogeneous and inhomogeneous parts by performing a spatial average, $\langle Q\rangle=\int Q \sqrt{g^{(3)}} d^{3} x / V$, where $Q$ is any quantity that is to be averaged, $g^{(3)}$ is the determinant of the three metric and $V$ is the volume of a finite box over which the integration is performed. Periodic boundary conditions are imposed on the box and the limit of $V$ approaching infinity is taken. Hence, the modified Friedmann equation becomes

$$
8 \pi G a^{2}\langle\rho\rangle=3\left(\frac{a^{\prime}}{a}\right)^{2}+\left\langle(\vec{\nabla} \phi)^{2}\right\rangle .
$$

The inhomogeneous part of the time-time equation is then the cosmological generalization of Poisson's equation, where the second order terms are obviously small compared to first order ones. Moreover, Poisson equation guarantees that the metric perturbation is equivalent to the Newtonian potential on small scales and remains small everywhere away from black holes. This equation is used to estimate the significance of the correction $\epsilon=\left\langle(\vec{\nabla} \phi)^{2}\right\rangle / 3 H_{0}^{2}\left(H_{0}=a^{\prime} / a\right.$ is the Hubble constant today $)$ to the Friedmann equation, given a realistic model of density fluctuations. The averaging can be rewritten in terms of the power spectrum as

$$
\epsilon=\frac{4 \pi}{3} \int k^{3} P_{\phi}(k)\left(k / H_{0}\right)^{2} d \ln k=3 \pi \int k^{3} P_{\delta}(k)\left(H_{0} / k\right)^{2} d \ln k .
$$

$4 \pi k^{3} P_{\delta}(k) \approx 1$ today is at $k^{-1} \approx 10 M p c$ from which follows $\epsilon \approx 10^{-5}$, provided that there is no divergence on very small scales. To investigate this possibility we use nonlinear evolution of realistic power spectra, using $\mathrm{N}$ body simulations. The conclusion from these studies is that the logarithmic contribution to $\epsilon$ peaks at the transition from linear to nonlinear scale and is in all models of the order of $10^{-5}$.

To conclude, we argue that linear metric perturbation theory in the gauge above provides an excellent description of clumpy universe today and the corrections to the Friedmann equation are found to be negligible. For light propagation, second order corrections to the geodesic equation (terms proportional to $\phi \phi_{, i}$ ) are necessarily small compared to the first order terms (those proportional to $\phi_{, i}$ ). The deflection of light ray is dominated by the linear terms and higher order terms are unimportant even in a clumpy universe, provided that the metric perturbations remain small.

\section{References}

T. Futamase, Phys. Rev. Lett. 61, 2175 (1988) 